\title{
Coverage and Timeliness of Combined Military and Veteran Surveillance Systems
}

\author{
Howard S. Burkom 1 , Yevgeniy Elbert ${ }^{1}$, Carla Winston², Julie Pavlin*3, Cynthia Lucero- \\ Obusan $^{2}$ and Mark Holodniy²
}

${ }^{1} J o h n s$ Hopkins University Applied Physics Laboratory, Laurel, MD, USA; ${ }^{2}$ Veterans Health Administration, Palo Alto, CA, USA;

${ }^{3}$ Armed Forces Health Surveillance Center, Silver Spring, MD, USA

\section{Objective}

We determined the utility and effective methodology for combining patient record information from the Departments of Veterans Affairs (VA) and Defense (DoD) health surveillance systems.

\section{Introduction}

An objective of the Joint VA/DoD BioSurveillance System for Emerging Biological Threats project is to improve situational awareness of the health of combined VA and DoD populations. DoD and VA both use versions of the Electronic Surveillance System for the Early Notification of Community-Based Epidemics (ESSENCE). With a retrospective outpatient data collection available, we analyzed relative coverage and timeliness of the two systems to understand potential benefits of a joint system.

\section{Methods}

We used the US Office of Management and Budget's core-based statistical area (CBSA) to group data from the respective systems by megapolitan ( $>1$ million), metropolitan (50,000-1 million) and micropolitan $(10,000-50,000)$ areas. We performed frequency analyses and mapped coverage of the VA and DoD medical systems in these CBSAs. To determine comparability, we compared International Classification of Diseases, 9th Revision (ICD-9) code usage from 2007-2010 by age group in the respective systems and then formulated a working definition of influenza-like illness (ILI). We then compared CBSA-level temporal detection timeliness in the two systems for the H3N2 epidemic of 2007-9 and the H1N1 pandemic in 2009.

\section{Results}

We identified a total of 939 CBSAs, with generally diffuse geographic coverage by VA facilities and higher concentration in larger metro and mega areas for DoD facilities. Of the 51 mega CBSAs, all have at least one VA facility and $63 \%$ have a DoD facility. Coverage is sparser for the metro CBSAs and lighter still for the micro CBSAs (Table 1). Although the VA coverage is greater, in many CBSAs with dual coverage, the DoD visit volume is comparable or greater. Patient age distribution differs sharply, with $>85 \%$ of the VA patients over 45 years of age compared to $22 \%$ of DoD patients. For all CBSAs, the overall VA/DoD visit ratio is 1.92 , but the ratios for $0-17$ years is $0.004,18-44$ years $0.33,45-64$ years 5.20 and $>65$ years 11.63 .

Based on an analysis of ICD-9 codes used in the two systems, the DoD uses symptom-based ILI codes far more frequently than the VA, especially codes for diseases often seen in children (e.g., otitis media). Analysis of ILI-related codes assigned in both systems led to a common code set for comparative analysis. From applying alerting algorithms to visit counts based on this code set, detection was better in DoD data for $57 \%$ and $77 \%$ of CBSAs for seasonal and pandemic influenza, respectively, and better in VA data for $37 \%$ and $14 \%$ of CBSAs (Table 2). The VA system performed better during the typical H3N2 seasonal flu compared to the H1N1 outbreak. The DoD system performed better during the H1N1 pandemic, although outperformed the VA for both.

\section{Conclusions}

The coverage analysis demonstrates two complementary surveillance systems with evident benefits to a fused national health picture. The VA system patient volume roughly doubles the DoD system, and provides better geographic coverage in smaller CBSAs; however, the DoD includes younger populations, better coverage in strategic metro areas, and more pre-diagnostic ILI coding. From analysis of both outbreaks, relative timeliness could be improved in $92 \%$ of CBSAs with access to both systems, with more information provided in CBSAs where only one type of facility exists.

Table 1. Counts of CBSAs containing VA and DoD facilities

\begin{tabular}{|c|c|c|c|}
\hline Coverage Summary & Pop $>1$ Million & $>50,000-1$ million & $>10,000-50,000$ \\
\hline Both & 32 & 94 & 6 \\
\hline Neither & 0 & 149 & 244 \\
\hline DoD Only & 0 & 16 & 5 \\
\hline VA only & 19 & 292 & 82 \\
\hline Visit Counts DoD>VA & 6 & 56 & 5 \\
\hline Visit Counts DoD<VA<5*DoD & 10 & 24 & 1 \\
\hline Visit Counts VA $>5 *$ DoD & 16 & 14 & 0 \\
\hline
\end{tabular}

Table 2. Relative timeliness by CBSAs with both DoD and VA facilities with a minimum average of 2 ILI visits/week

\begin{tabular}{|c|c|c|c|}
\hline Alert Timeliness & $07-08 \mathrm{H} 3 \mathrm{~N} 2$ & $09 \mathrm{H} 1 \mathrm{~N} 1$ & Both \\
\hline Same Week & $6(6 \%)$ & $5(5 \%)$ & $11(6 \%)$ \\
\hline DoD Earlier & $51(55 \%)$ & $60(65 \%)$ & $111(60 \%)$ \\
\hline VA Earlier & $33(35 \%)$ & $10(11 \%)$ & $43(23 \%)$ \\
\hline DoD Only & $2(2 \%)$ & $12(13 \%)$ & $14(8 \%)$ \\
\hline VA Only & $1(1 \%)$ & $3(3 \%)$ & $4(2 \%)$ \\
\hline No Alert & 0 & $3(3 \%)$ & $3(2 \%)$ \\
\hline
\end{tabular}

\section{Keywords}

Syndromic surveillance; Merged systems; Government

\section{*Julie Pavlin}

E-mail: julie.pavlin@us.army.mil 